\title{
Measurement of the top quark mass and width with CDF detector
}

\section{Hyun Su Lee* On Behalf of the CDF Collaboration}

Enrico Fermi Institute, University of Chicago

E-mail: hsleedfnal.gov

\begin{abstract}
The top quark mass and width are fundamental parameters of the Standard Model (SM). The precision measurement of top quark mass combined with $\mathrm{W}$ boson mass measurement can constrain the mass range of the SM Higgs boson, which is the only unobserved SM particle. The precision measurement of top quark width is a good test of SM because the SM prediction is very precise. In this letter we present updated top quark mass and width measurement results of selected analyses using data up to $5.6 \mathrm{fb}^{-1}$ of $p \bar{p}$ collisions at Tevatron Fermilab obtained by the CDF detector.
\end{abstract}

35th International Conference of High Energy Physics - ICHEP2010,

July 22-28, 2010

Paris France

${ }^{*}$ Speaker. 


\section{Introduction}

The top quark, observed by both the CDF and D0 experiments in 1995 [1, 2], is by far the heaviest known elementary particle and its mass is almost 40 times heavier than its isospin partner, the bottom $(b)$ quark [3]. Due to the heavy mass, the top quark plays an important role in electroweak radiative corrections. Therefore, top quark mass $\left(\mathrm{M}_{\text {top }}\right)$ measurements are important tests of the Standard Model (SM) and provide constraints on the Higgs boson mass. Its large mass results in the largest decay width and hence the shortest lifetime. A precise measurement of top quark width $\left(\Gamma_{\text {top }}\right)$ is another important test of the SM, whose prediction has a precision of order $1.5 \%$ [4].

\section{Top quark production and decay}

Top quarks at the Tevatron are predominantly produced in pairs, and decay almost always to a $\mathrm{W}$ boson and a $b$ quark in the SM. The topology of $t \bar{t}$ events depends on the different decay of the two $\mathrm{W}$ bosons. In the dilepton channel, each $\mathrm{W}$ boson decay to charged lepton (electron and muon) and neutrino. Events in this channel thus contain two leptons, two $b$-quark jets, and two undetected neutrino. Because of the presence of two leptons, this channel has the lowest background. However the dilepton channel has the smallest branching fraction. In the all-jets channel, each $\mathrm{W}$ boson decays to two jets so that this channel contains two $b$ quark jets and four light quark jets. This channel has the largest branching fraction but also the largest background from QCD multijet production. The lepton+jets channel has one $\mathrm{W}$ boson decaying leptonically and the other hadronically so that we have one charged lepton, two $b$-quark jets, two light quark jets, and one undetected neutrino. Because of the relatively large branching fraction with manageable background levels, we made the most precise $\mathrm{M}_{\text {top }}$ and $\Gamma_{\text {top }}$ measurements using events in the lepton+jets decay topology.

To improvement the $\mathrm{M}_{\text {top }}$ and $\Gamma_{\text {top }}$ measurement, CDF collaboration identify $b$ quarks using the properties of the longer lifetime of metastable B hadrons [5]. Therefore jets arising from $b$ quarks have secondary vertices that are displayed from the primary collision vertex. $b$-tagging significantly improve not only background fraction but also the combinatorics of jet-to-parton assignments, improving $\mathrm{M}_{\text {top }}$ resolution.

In the lepton+jets and all-jets channels, we have at least one $\mathrm{W}$ boson decaying hadronically (W decaying to two jets). Therefore we use the reconstructed dijet mass from $\mathrm{W}$ boson decay to constrain, in situ, the largest systematic in $\mathrm{M}_{\text {top }}$ measurements, the jet energy scale (JES), which is the calibration between jets energies and parton level energies, because of the narrow decay width and well known mass of the $\mathrm{W}$ boson.

\section{Top quark mass measurement}

For the $\mathrm{M}_{\text {top }}$ measurements, two primary techniques have been established. The template method (TM) uses the distributions of variables (templates) which are strongly correlated with the top quark mass and JES. In the building of a probability, only a few variables (usually less than two) are used, for instance reconstructed top quark mass and dijet mass in the lepton+jets channel. The 
Matrix Element Method (ME) uses event's probability to be a combinates signals and background. ME exploit all the information in the event by using a leading order matrix element calculation convoluted with parton distribution function and transfer functions (TFs) making connection between detector response and parton level particle. Because we can use all the information in principle, ME usually provide better precision of $\mathrm{M}_{\text {top }}$ than $\mathrm{TM}$. Both techniques employ a likelihood to compare data to the modeling of signals and background to extract $\mathrm{M}_{\mathrm{top}}$.

CDF has a ME based measurement in the lepton+jets channel using $5.6 \mathrm{fb}^{-1}$. This analysis integrates over more than 19 variables using a quasi-MC integration technique to account imperfect assumptions about perfectly measured angles and intermediate particle masses. TF is parameterized as a function of $\eta$ and $p_{T}$ separately for $b$-jets and light jets. This analysis also makes a cut using a NN to reject not only background contribution but also poorly modeled signal events where the objects in the detector do not match the assumed partons at the matrix element level. With in situ JES calibration, we measure $\mathrm{M}_{\text {top }}=173.0 \pm 1.2 \mathrm{GeV} / c^{2}$ [6]. This measurement is the most precise top quark mass measurement in the world to date.

CDF has another lepton+jets channel measurement using TM. We uses $4.8 \mathrm{fb}^{-1}$ of $p \bar{p}$ collisions. In this measurement, three variables are used to estimate probabilities of events. The first two variables are the reconstructed top quark mass from the kinematic fitter [7] and dijet mass from hadronically decaying $\mathrm{W}$ boson used to make the $\mathrm{M}_{\mathrm{top}}$ measurement with in situ JES calibration in the same channel [8]. In addition, a $3^{\text {rd }}$ variable is introduced: the $2^{\text {nd }}$ best reconstructed top quark mass by choosing $2^{\text {nd }}$ jets to parton assignment based on the $\chi^{2}$ of kinematic fitter. To take into account correlation between the variables and build probabilities without parameterization, kernel density estimation (KDE) [8 9] was employed. This revisit in a measured $\mathrm{M}_{\text {top }}$ $=172.0 \pm 1.5 \mathrm{GeV} / c^{2}$ [10]. This measurement use a technique complementary to the ME based measurement and gives a consistent result.

CDF has a dilepton channel measurement using TM using same amount of data with Lepton+jets channel. Two variables sensitive to $\mathrm{M}_{\text {top }}$ are used by taking into account the correlations using KDE. One variable is the reconstructed top quark mass using the neutrino weighting algorithm (NWA) [11 12] in the underconstrained system from two neutrinos. The unknown pseudorapidities of the two neutrinos are integrated over. The solutions for a given top quark mass are weighted by using measured missing transverse energy. The other variable is $m_{T 2}$ [13, 14] which is a measure of transverse mass in two missing particles final states. It provides a measured $\mathrm{M}_{\text {top }}$ in the dilepton channel [15] and is the first use of this technique. The simultaneous measurement with the two variables gives $\mathrm{M}_{\text {top }}=170.6 \pm 3.8 \mathrm{GeV} / \mathrm{c}^{2}$ 《10].

Because two TM measurements share the same machinery, a simultaneous measurement can be made using the lepton+jets and dilepton channels [8]. The correlation of systematic uncertainties is intrinsically taken into account. The combined measurement both lepton+jets and dilepton channels using $4.8 \mathrm{fb}^{-1}$ data gives $\mathrm{M}_{\mathrm{top}}=171.9 \pm 1.5 \mathrm{GeV} / \mathrm{c}^{2}$ [10].

The measurements from different channels are combined using the best linear unbiased estimation technique. As an average of CDF measurements, we have $\mathrm{M}_{\mathrm{top}}=173.1 \pm 1.2 \mathrm{GeV} / c^{2}$ [16]. By combining with D0 results [17], we have $\mathrm{M}_{\text {top }}=173.3 \pm 1.1 \mathrm{GeV} / c^{2}$ 《18 as a world average.

\subsection{Top and anti-top quark mass difference measurement}

The precision determination of $\mathrm{M}_{\text {top }}$ allows us to measure the mass difference between top 
quark and anti-top quark to a few $\mathrm{GeV}$. In the CPT theorem, which is fundamental to any local Lorentz-invariant quantum field theory, the quark mass should be same as its anti-quark partner. Despite the fact that no violations have ever been observed in the meson and baryon sectors, it is important to test CPT violation in all sectors such as quarks and high mass particles. CDF collaboration measure the mass difference between top quark and anti-top quark $\left(\delta \mathrm{M}_{\text {top }}\right)$ in the lepton+jets channel using the TM technique. We reconstruct the mass difference using modificed kinematic fitter allowing mass difference between hadronic top quark and leptonic top quark. Using $5.6 \mathrm{fb}^{-1}$ of $p \bar{p}$ collisions, we measure $\delta \mathrm{M}_{\mathrm{top}}=-3.3 \pm 1.7 \mathrm{GeV} / c^{2}$ [19]. It is consistent with CPT symmetry at a $2 \sigma$ level and also consistent with the published result from D0 Collaboration [20]. This is the most precise measurement of a quark and anti-quark mass difference.

\section{Top quark width measurement}

We measure the top quark width using $4.3 \mathrm{fb}^{-1}$ of $p \bar{p}$ collision using TM technique. The $\mathrm{M}_{\text {top }}$ and the mass of $\mathrm{W}$ boson that decays hadronically are reconstructed for each event and compared with templates of different $\Gamma_{\text {top }}$ and deviations from nominal jet energy scale $\left(\Delta_{\mathrm{JES}}\right)$ to perform a simultaneous fit for both parameters, where $\Delta_{\mathrm{JES}}$ is used for the in situ calibration of the jet energy scale. By applying a Feldman-Cousins approach, we establish an upper limit at $95 \%$ confidence level of $\Gamma_{\text {top }}<7.6 \mathrm{GeV}$ and a two-sided $68 \% \mathrm{CL}$ interval of $0.3 \mathrm{GeV}<\Gamma_{\text {top }}<4.4 \mathrm{GeV}$ [21]. This result supports top quark decay before hadronization.

\section{Conclusion}

The CDF collaboration has performed a robust set of analyses using many techniques and improvements to have better understand the important fundamental parameter of the SM. As a result, CDF ME measurement gives $\mathrm{M}_{\mathrm{top}}=173.0 \pm 1.2 \mathrm{GeV} / c^{2}$ contributing to a new world average, $\mathrm{M}_{\mathrm{top}}=173.3 \pm 1.1 \mathrm{GeV} / c^{2}$, and TM measurement gives $\Gamma_{\text {top }}<7.6 \mathrm{GeV}$. By end of Run II, we expect $8 \sim 12 \mathrm{fb}^{-1}$ of data delivered by the Tevatron which could be almost a double the data sample used in this letter. An ultimate precision of about $\mathrm{M}_{\text {top }}$ less than $1 \mathrm{GeV} / c^{2}$ and two sigma bound of $\Gamma_{\text {top }}$ will be possible.

\section{Acknowledgments}

I would like to thank for the CDF colleagues for their efforts to carry out these challenging physics analyses. I also thank for the conference organizers for a very rich week of physics.

\section{References}

[1] F. Abe et. al. (CDF Collaboration), Phys. Rev. Lett. 74, 2626 (1995).

[2] S. Abachi et. al. (D0 Collaboration), Phys. Rev. Lett. 74, 2632 (1995).

[3] C. Amsler et. al. (Particle Data Group), Phys. Lett. B 667, 551 (2008).

[4] M. Jezabek and J. H. Kuhn, Phys. Rev. D 48, 1910 (1993); M. Jezabek and J. H. Kuhn, Nucl. Phys. B 1, 1989 (.) 
[5] D. Acosta et. al. (CDF Collaboration), Phys. Rev. D 71, 052003 (2005).

[6] CDF Collaboration, CDF Conference note 10191 (2010).

[7] A. Abulencia el. al. (CDF Collaboration), Phys. Rev. D 73, 032003 (2006).

[8] T. Aaltonen et. al. (CDF Collaboration), Phys. Rev. D 79, 092005 (2009).

[9] K. Cranmer, Comput. Phys. Commun. 136, 198 (2001), arXiv:hep-ex/0011057v1.

[10] CDF Collaboration, CDF Conference note 10033 (2010).

[11] B. Abbott et. al. (D0 Collaboration), Phys. Rev. D 60, 052001 (1999).

[12] A. Abulencia et. al. (CDF Collaboration), Phys. Rev. D 73, 112006 (2006).

[13] C. Lester and D. Summers, Phys. Lett. B 463, 99 (1999).

[14] A. Barr, C Lester, and P. Stephens, J. Phys. G 29, 2343 (2003).

[15] T. Aaltonen el. al. (CDF Collaboration), PRD 81, 031102 (2010).

[16] CDF Collaboration, CDF Conference note 10202 (2010).

[17] D0 Collaboration, Do Conference note 5900 (2009).

[18] CDF and D0 Collaboration, FERMILAB-TM-2466-E (2009), CDF Conference note 10210 (2010), DO Conference note 6090 (2010), arXiv:1007.3178v1.

[19] CDF Collaboration, CDF Conference note 10173 (2010).

[20] V. M. Abazov el. al. (D0 Collaboration), Phys. Rev. Lett. 103, 132001 (2009).

[21] T. Aaltonen el. al. (CDF Collaboration), arXiv:1008.3891v1, CDF Conference note 10035 (2010). 